\title{
ORIGINAL
}

\section{INCIDENCIA DE LA HIPERTRANSAMINEMIA MARCADA EN UN DEPARTAMENTO DE SALUD DE LA COMUNIDAD VALENCIANA (PERÍODO 2002-2003)}

\author{
Esther Castillo-Gómez (1), Javier Colomina-Rodríguez (1,2), Flor Gimeno-Vilarrasa (1), María \\ Jesús Moll-Navarro (1) y Antonio Guerrero-Espejo (1,2).
}

(1) Unidad de Investigación y Docencia. Hospital de La Ribera. Alzira, Valencia.

(2) Servicio de Microbiología. Hospital de La Ribera. Alzira, Valencia.

\section{RESUMEN}

Fundamento: La hipertransaminasemia (HT) marcada es una situación no infrecuente en la práctica clínica, que suele interpretarse como lesión hepática aguda primaria. Los objetivos del estudio fueron determinar la incidencia, tasa de mortalidad y etiología de la HT marcada en la población general, y su utilidad como indicadora de daño hepático agudo primario.

Métodos: Se realizó un estudio retrospectivo los pacientes con HT marcada (ALT $>400 \mathrm{U} / \mathrm{L}$ ) atendidos durante un periodo de 2 años en los centros sanitarios del Departamento 11 de la Comunidad Valenciana. Se revisaron las historias clínicas informatizadas y los resultados de los diversos exámenes complementarios efectuados, analizándose diversas variables: diagnóstico clínico y evolución, otros parámetros bioquímicos de función hepática, y marcadores de serología infecciosa y autoinmunidad.

Resultados: Se identificaron 414 pacientes con HT marcada (incidencia de 88 casos/100.000 hab./año), de los cuales 73 fallecieron (tasa de mortalidad de 16 fallecidos/100.000 hab/año). De las veinte etiologías encontradas, las más frecuentes fueron la colestasis extrahepática $(28,3 \%)$, la hepatopatía hipóxica $(14,6 \%)$ y la sepsis $(11,9 \%)$. El valor predictivo positivo de la HT marcada como indicador de lesión hepática aguda primaria fue del $27,7 \%$.

Conclusiones: La HT marcada es una alteración de notable incidencia en la población general y que lleva asociada una elevada tasa de mortalidad. Su etiología es muy diversa, aunque predomina la de origen extrahepático.

Palabras clave: Hipertransaminasemia. Colestasis extrahepática.

Correspondencia:

Javier Colomina Rodríguez.

Área de Diagnóstico Biológico.

Hospital de La Ribera

Ctra. Corbera Km 1, Alzira

46600 Valencia.

Correo electrónico: jcolomina@hospital-ribera.com

\section{ABSTRACT}

\section{Marked Hypertransaminasemia Incidence in a Health Department in the Autonomous Community of Valencia, Spain (2002-2003)}

Background: Marked hypertransaminasemia (HT) is not an infrequent situation within clinical practice, which is usually interpreted as primary acute liver damage. The objectives of this study were to determine the incidence, mortality rate and aetiology of marked HT among the general population, and the use therefore as an indicator of primary acute liver damage.

Methods: A retrospective study was made of all patients with marked HT (ALT>400 IU/L) which were attended over a two-year period at the Healthcare Department $n^{\circ} 11$ in the Autonomous Community of Valencia. The computerized medical records and the results of the different supplementary examinations made were reviewed, and an analysis was made of different variables: clinical diagnosis and evolution, other liver function-related biochemical parameters and autoimmune and infection serology markers.

Results: A total of 414 patients with marked HT were identified (incidence of 88 cases/100,000 inhab./year), 73 of whom died (mortality rate of 16 deaths/100,000 inhab./year). Of the twenty aetiologies found, the most frequent were extrahepatic cholestasis $(28.3 \%)$, hypoxic hepatopathy $(14.6 \%)$ and sepsis $(11.9 \%)$. The positive predictive value of marked HT as an indicator of primary acute liver damage was $27.7 \%$.

Conclusions: Marked HT is a disorder having a remarkable incidence rate among the general population, entailing a high mortality rate. Its aetiology is widely varied, being however the extrahepatic origin predominant.

Key words: Hypertransaminasemia. Hepatic cholestasis. 


\section{INTRODUCCIÓN}

La elevación de los niveles de transaminasas en sangre o hipertransaminasemia es una situación que se presenta con considerable frecuencia en la práctica clínica $^{1}$. La alanina aminotransferasa (ALT), también conocida como transaminasa glutámico pirúvica (GPT), es una enzima citosólica que se localiza preferentemente en hígado, por lo que el aumento de su nivel en suero es un indicador sensible de daño hepatocelular. Sin embargo, la especificidad de este indicador no es del todo absoluta, ya que también existen bajas concentraciones de esta proteína en músculo esquelético, riñón y corazón, pudiéndose producir elevaciones de ALT en ausencia de lesión celular hepática ${ }^{2}$.

En general, la magnitud de la hipertransaminasemia (HT) no es una herramienta útil para evaluar el grado del daño hepatocelular, aunque si puede orientarnos sobre su etiología. El origen de las HT consideradas leves (ALT <200 U/L) o moderadas (ALT 200-400 U/L) en ocasiones es difícil de determinar, ya que ambas pueden tener lugar en contextos clínicos muy diversos, tanto de afectación hepática (hepatitis crónica, hepatitis aguda subclínica o en regresión, enfermedad biliar), como extrahepática (enfermedad celiaca, patologías musculares o tiroideas, entre otros). La HT marcada $($ ALT $>400 \mathrm{U} / \mathrm{L})$ raramente observada en procesos en los que el hígado no resulta afectado (rabdomiolisis, miopatía) ${ }^{3,4}$, indica con elevada frecuencia lesión hepatocelular. Es habitualmente interpretada por el clínico como un indicador de lesión hepática aguda primaria (hepatitis viral, medicamentosa, etc.), sin embargo, esta percepción no siempre es correcta ya que puede ser secundaria a otros procesos que derivan en un daño celular hepático agudo (hepatopatía hipóxica, colestasis extrahepática, etc.).
Los objetivos del estudio fueron conocer la importancia de la HT marcada en un Área Sanitaria en términos de incidencia y tasa de mortalidad, así como establecer su etiología y el grado de utilidad a la hora de interpretar esta alteración como indicadora de lesión hepática aguda primaria.

\section{SUJETOS Y MÉTODOS}

Se realizó un estudio retrospectivo de los pacientes con HT marcada atendidos entre enero de 2002 y diciembre de 2003 en los centros de atención primaria y especializada del Departamento de Salud ${ }^{\circ} 11$ de la Comunidad Valenciana (234.139 habitantes).

Los niveles de ALT se determinaron mediante métodos convencionales utilizando un autoanalizador Hitachi $917^{\circledR}$ (Roche $)^{5}$. Las muestras con valores $>400 \mathrm{U} / \mathrm{L}$ fueron reanalizadas. En caso de presentar signos de posibles interferencias (hemólisis, lipemia, etc) se solicitó una nueva muestra.

En los pacientes con HT marcada se revisaron las historias clínicas informatizadas y los resultados de los exámenes complementarios efectuados, analizándose diversas variables: diagnóstico clínico y evolución, otros parámetros bioquímicos de función hepática (aspartato aminotransferasa, gamma-glutamil transpeptidada, fosfatasa alcalina, bilirrubina), y marcadores de serología infecciosa y autoinmunidad (virus de las hepatitis A, B y C, citomegalovirus, virus de Epstein-Barr, virus de la inmunodeficiencia humana, toxoplasma y anticuerpos antinucleares). En caso de existir, también se analizaron los informes de anatomía patológica y radiología. Los casos de los que no se disponía de suficiente información clínica fueron excluidos. 
Para la clasificación etiológica de la HT marcada se establecieron distintos grupos y subgrupos teniendo en cuenta las clasificaciones internacionales vigentes. Se consideró etiología no filiada cuando en la historia clínica no existían evidencias para atribuir la elevación de ALT a una causa determinada.

Los datos recogidos se analizaron mediante estadística descriptiva univariada, realizándose además análisis estratificados por grupos de edad, grupos diagnósticos y sexo. También se realizaron análisis de varianzas para detectar diferencias significativas entre los grupos diagnósticos, tanto en los niveles de ALT como en la edad de los pacientes. Para conocer la utilidad diagnóstica del indicador ALT $>400$ U/L se calculó el valor predictivo positivo para la lesión hepática aguda primaria ${ }^{6}$, excluyéndose del cálculo a todos los pacientes con etiología no filiada. Los cálculos estadísticos se realizaron con el programa SPSS 12.0 para Windows.

\section{RESULTADOS}

Durante el periodo analizado se identificaron 414 personas con HT marcada, lo que supone una incidencia de 88 casos por cada 100.000 habitantes y año. Se excluyó a 18 pacientes por documentación clínica insuficiente, quedándose la población de estudio en 396 pacientes, con 226 hombres $(57,1 \%)$ y 170 mujeres $(42,9 \%)$, y una edad media de $56 \pm 21$ años (mediana: 60 años; rango: 1-94 años). El 2,8\% de los pacientes estudiados eran niños menores de 14 años, el $53,7 \%$ adultos y el 43,5\% mayores de 64 años.

Los resultados de las pruebas bioquímicas de función hepática se recogen en la tabla 1. Cabe destacar que un elevado porcentaje $(96,2 \%)$ de pacientes también mostraba niveles elevados de AST.

Se identificó un total de 20 etiologías distintas (grupos diagnósticos) para la HT marcada. La frecuencia de cada grupo se muestra en la figura 1. La frecuencia con la que la elevación de ALT se debió a una lesión hepática aguda primaria fue globalmente del 26,3\%. En general, la etiología más frecuente fue la colestasis extrahepática $(28,3 \%)$, seguida de la hepatopatía hipóxica $(14,6 \%)$ y la sepsis $(11,9 \%)$. En el grupo de las colestasis el $85,8 \%$ de los pacientes presentaron obstrucción por litiasis o pancreatitis aguda, siendo el resto de causas poco o muy poco frecuentes (figura 2). Algo similar ocurre en el grupo de las hepatopatías hipóxicas, donde más de la mitad de los casos

Tabla 1

Resultados de las pruebas de función hepática realizadas en 396 pacientes que presentaron hipertransaminasemia marcada (ALT $>400 \mathrm{U} / \mathrm{L}$ ) durante los años 2002-2003 en el Departamento de Salud no 11 de la Comunidad Valenciana

\begin{tabular}{|l|c|c|c|}
\hline Parámetro & Media \pm DS & Rango & Pacientes con valores >2N \\
\hline ALT $(\mathrm{U} / \mathrm{L})$ & $1.009 \pm 53$ & $(402-11.680)$ & $396(100 \%)$ \\
\hline AST $(\mathrm{U} / \mathrm{L})$ & $1.117 \pm 99$ & $(110-17.780)$ & $381(96,2 \%)$ \\
\hline GGT $(\mathrm{U} / \mathrm{L})$ & $341 \pm 25$ & $(6-4.032)$ & $192(48,5 \%)$ \\
\hline FA $(\mathrm{U} / \mathrm{L})$ & $243 \pm 16$ & $(27-3.506)$ & $85(21,5 \%)$ \\
\hline BT $(\mathrm{mg} / \mathrm{dL})$ & $3,36 \pm 0,24$ & $(0,18-34,12)$ & $146(36,9 \%)$ \\
\hline BD $(\mathrm{mg} / \mathrm{dL})$ & $2,43 \pm 0,21$ & $(0-26)$ & $205(51,8 \%)$ \\
\hline BI $(\mathrm{mg} / \mathrm{dL})$ & $0,95 \pm 0,06$ & $(0,01-8,12)$ & $49(12,4 \%)$ \\
\hline
\end{tabular}

DS: desviación estándar. N: valor normal. ALT: alanina aminotransferasa. AST: aspartato aminotransferasa: GGT: gamma-glutamil transpeptidada: FA: fosfatasa alcalina: BT: bilirrubina total. BD: bilirrubina directa. BI: bilirrubina indirecta. 


\section{Figura 1}

Etiologías de HT marcada. Con un asterisco (*) se indican aquellas patologías que provocan una lesión hepática aguda primaria

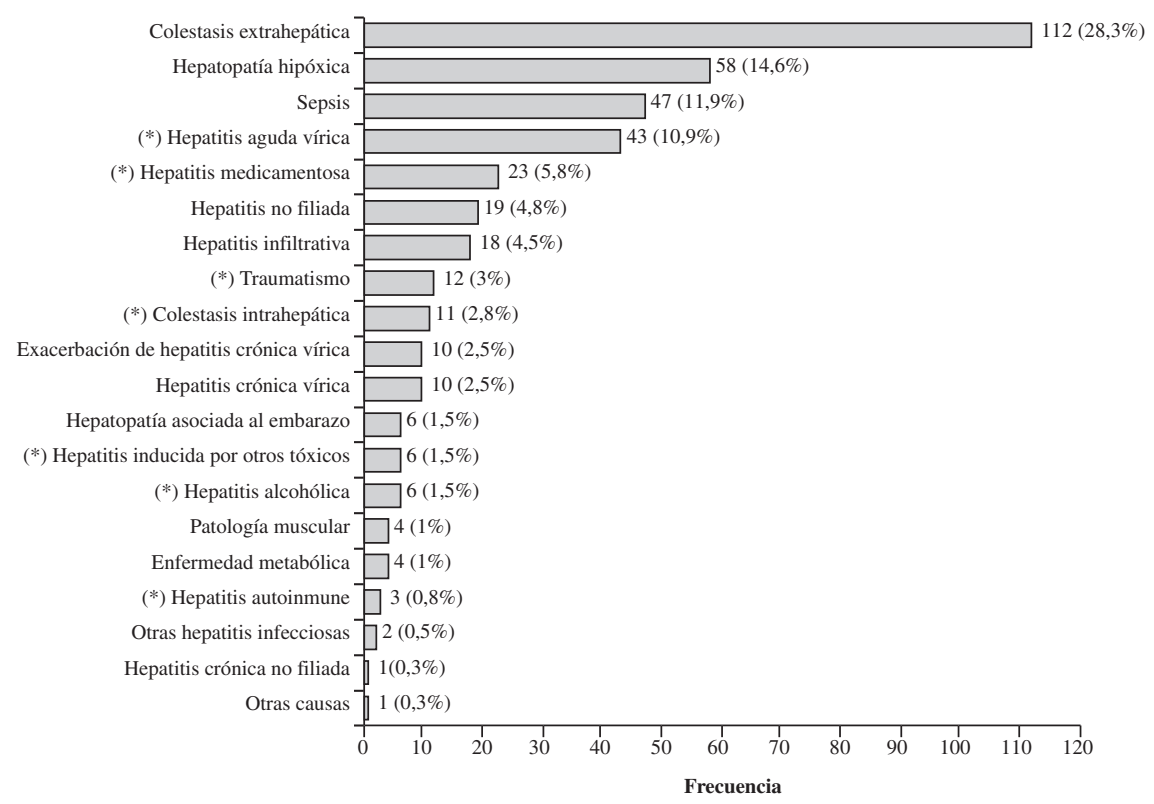

Figura 2

Causas de colestasis xtrahepática en pacientes $(n=112)$ con HT marcada

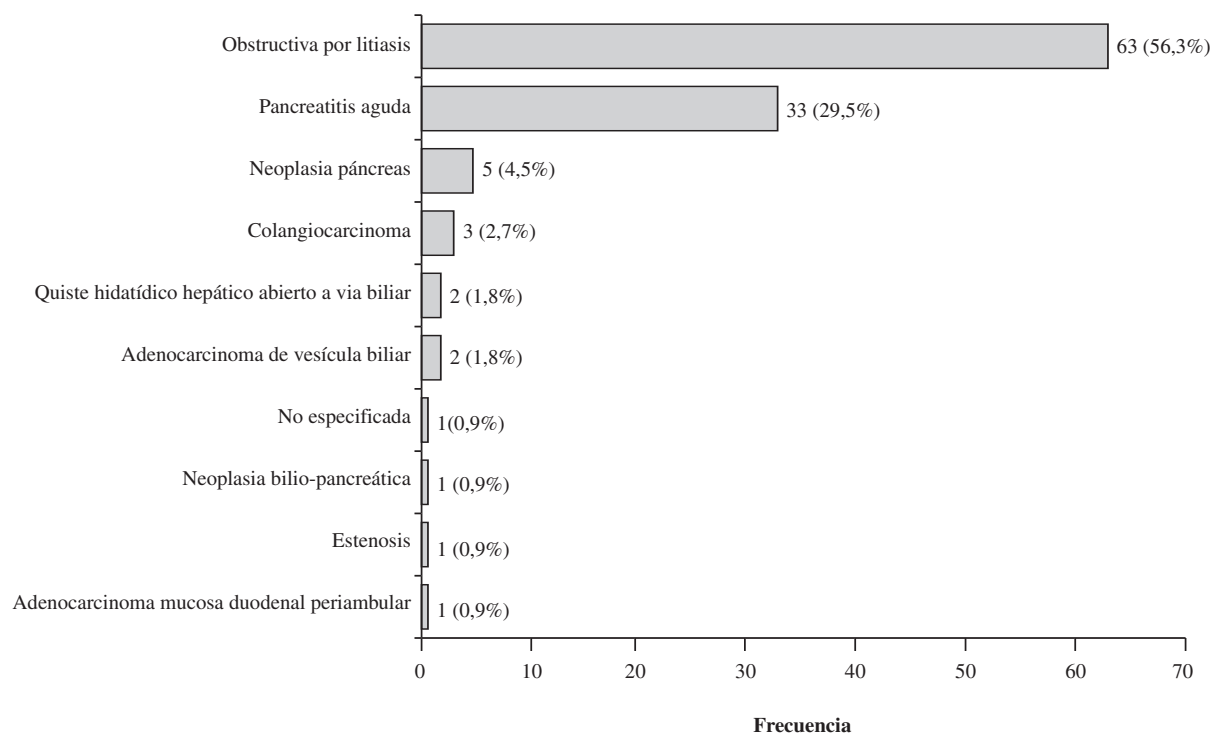


Figura 3

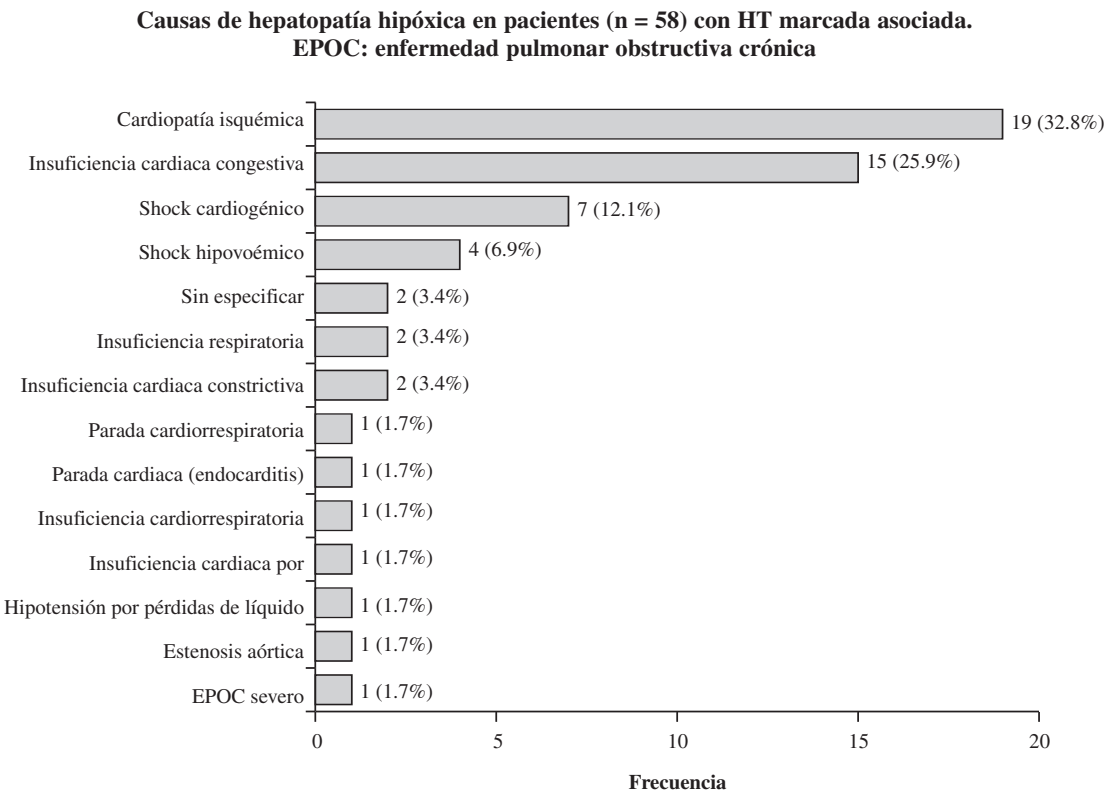

$(58,7 \%)$ se deben a una cardiopatía isquémica o a una insuficiencia cardiaca congestiva (figura 3).

El mayor valor medio de ALT (2.039 U/L) correspondió al grupo de la hepatitis inducidas por drogas y/o tóxicos industriales, mientras que el valor más bajo (460 U/L) se asoció con las patologías musculares. El grupo de pacientes con hepatopatía hipóxica fue el de mayor edad media (72 años), y el de la hepatopatía asociada al embarazo el de menor (29 años).

En los grupos diagnósticos más frecuentes el análisis estadístico de la variable ALT reveló diferencias significativas entre el grupo de pacientes con colestasis extrahepática y los grupos de hepatitis agudas víricas $(\mathrm{p}=0,033)$, sepsis $(\mathrm{p}=0,000)$ y hepatopatía hipóxica $(\mathrm{p}=0,000)$ (tabla 2). Este último, también mostró diferencias con respecto al grupo de las hepatitis no filiadas $(\mathrm{p}=0,043)$ pero no respecto a la hepatopatía infiltrativa $(\mathrm{p}=0,183)$. En cuanto a la edad media de los pacientes, se detectaron diferencias estadísticas entre muy diversos grupos diagnósticos (tabla 2).

Las etiologías variaron en función del grupo de edad pero no en función del sexo. En los niños la más frecuente fue la hepatitis viral aguda $(63,6 \%)$, mientras que en los adultos predominaron la colestasis extrahepática $(26 \%)$ y la hepatitis viral aguda $(14,6 \%)$. En las personas mayores de 64 años las causas más detectadas fueron la colestasis extrahepática $(33,1 \%)$ y la hepatopatía hipóxica $(27,9 \%)$.

El valor predictivo positivo de la ALT $>400$ U/L para la lesión hepática aguda primaria fue del $27,7 \%$.

Setenta y tres pacientes $(18,4 \%)$ fallecieron durante el episodio de HT marcada, lo que supone una tasa de mortalidad asociada a esta patología de 16 fallecidos por cada 100.000 habitantes y año. La sepsis fue el grupo diagnóstico con mayor por- 
Tabla 2

Significación estadística de las variables de valor medio de alanino aminotransferasa (ALAT) y de edad media de los pacientes en los grupos diagnósticos más frecuentes

\begin{tabular}{|c|c|c|c|c|c|c|c|c|c|c|c|c|}
\hline Variable & \multicolumn{12}{|c|}{ ALAT } \\
\hline \multirow{12}{*}{$\begin{array}{l}\text { D } \\
\text { A } \\
\text { D }\end{array}$} & & A & B & C & D & $\mathbf{E}$ & F & G & H & I & $\mathbf{J}$ & $\mathbf{K}$ \\
\hline & A & & 0,000 & 0,000 & 0,033 & 0,914 & 1,000 & 1,000 & 0,999 & 1,000 & 1,000 & 1,000 \\
\hline & B & 0,069 & & 1,000 & 0,863 & 0,376 & 0,043 & 0,183 & 0,568 & 0,203 & 0,136 & 0,160 \\
\hline & C & 1,000 & 0,478 & & 0,999 & 0,823 & 0,230 & 0,555 & 0,879 & 0,504 & 0,374 & 0,417 \\
\hline & D & 0,000 & 0,000 & 0,000 & & 0,996 & 0,678 & 0,930 & 0,994 & 0,858 & 0,742 & 0,782 \\
\hline & E & 0,060 & 0,000 & 0,075 & 0,002 & & 0,998 & 1,000 & 1,000 & 0,999 & 0,994 & 0,996 \\
\hline & F & 0,000 & 0,000 & 0,001 & 0,348 & 0,964 & & 1,000 & 1,000 & 1,000 & 1,000 & 1,000 \\
\hline & G & 0,999 & 0,205 & 0,995 & 0,000 & 0,879 & 0,169 & & 1,000 & 1,000 & 1,000 & 1,000 \\
\hline & H & 0,001 & 0,000 & 0,001 & 0,950 & 0,803 & 1,000 & 0,089 & & 1,000 & 1,000 & 1,000 \\
\hline & I & 0,903 & 1,000 & 0,974 & 0,000 & 0,040 & 0,001 & 0,746 & 0,001 & & 1,000 & 1,000 \\
\hline & $\mathbf{J}$ & 0,022 & 0,000 & 0,022 & 0,744 & 0,989 & 1,000 & 0,391 & 1,000 & 0,009 & & 1,000 \\
\hline & $\mathbf{K}$ & 0,037 & 0,000 & 0,036 & 0,644 & 0,996 & 1,000 & 0,481 & 1,000 & 0,014 & 1,000 & \\
\hline
\end{tabular}

Los valores en negrita indican significación estadística $(\mathrm{p}<0,05)$. A: colestasis extrahepática. B: hepatopatía hipóxica. C: sepsis. D: hepatitis vírica aguda. E: hepatitis medicamentosa. F: hepatitis no filiada. G: hepatitis infiltrativa. H: traumatismo. I: colestasis intrahepática. J: exacerbación hepatitis vírica crónica. K: hepatitis vírica crónica.

\section{Figura 4}

Principales causas asociadas a mortalidad en pacientes con HT marcada

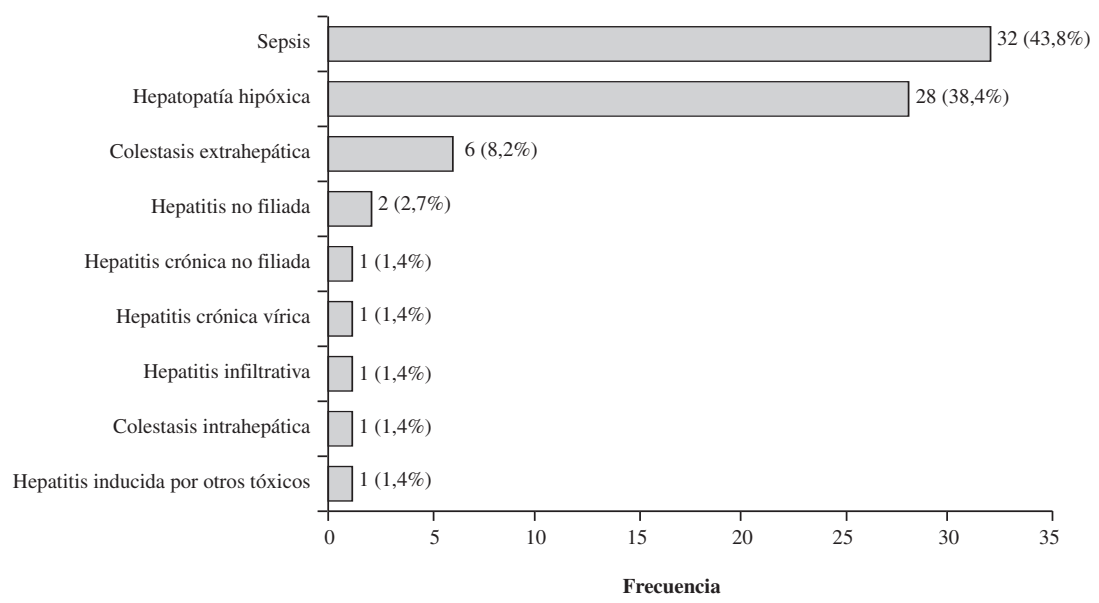

centaje de fallecidos $(43,8 \%)$, seguido del grupo de la hepatopatía hipóxica $(38,4 \%)$. En la colestasis extrahepática, la etiología más frecuente de HT marcada en el estudio, el porcentaje fue más bajo $(5,4 \%)$ (figura 4).

\section{DISCUSIÓN}

El presente estudio muestra que algo menos de 1 caso por 1.000 habitantes y año presentan HT marcada (concretamente 88 casos/100.000 habitantes/año) y que la 
mortalidad asociada puede llegar a alcanzar a casi la quinta parte de los que la padecen (16 fallecidos/100.000 habitantes/año en nuestra Área de Salud). Esta incidencia se aproxima a la detectada para la apendicitis aguda en nuestra zona geográfica (96 casos/100.000 habitantes/año) ${ }^{7}$. Su mortalidad es, sin embargo, superior a la detectada para la mayoría de las enfermedades infecciosas graves en países desarrollados, ya que por ejemplo la mortalidad de la neumonía infecciosa está alrededor del 5\% en pacientes inmunocompetentes ${ }^{8}$.

Durante años la HT marcada fue atribuida principalmente a las hepatitis víricas agudas ${ }^{9}$. En la última década, como consecuencia de la introducción de vacunas, el análisis de las reservas de sangre y la mejora de las condiciones higiénico sanitarias, la incidencia de las hepatitis virales ha disminuido considerablemente en los países desarrollados, haciendo que en la actualidad sean otras las etiologías que más comúnmente causan esta alteración en los niveles de transaminasas ${ }^{1}$.

Los resultados obtenidos demuestran que la elevación marcada de ALT puede tener etiologías muy diversas y que, al contrario de lo que se esperaba, se origina más frecuentemente por patologías que afectan secundariamente al hígado que por afecciones que causan un daño hepatocelular agudo primario. Es más, las tres etiologías de HT marcada más frecuentes (colestasis extrahepática, hepatopatía hipóxica y sepsis) no son primariamente hepáticas. Estos hallazgos son coincidentes con los de dos estudios recientes $^{10,11}$, en los que la hepatopatía hipóxica y las enfermedades pancreático-biliares fueron las dos etiologías predominantes. Las principales diferencias del presente estudio con respecto a otros radican en el mayor tamaño muestral, la mayor diversidad de etiologías atribuidas a la HT marcada (figura 1) y en la detección de algunas otras causas asociadas a ésta cuya aparición es relativamente infrecuente, como son el golpe de calor ${ }^{12}$, la rabdomiolisis $^{13}$, el síndrome de HELLP ${ }^{14}$ y la gastroenteritis viral con repercusión hepática ${ }^{15}$.

El valor predictivo positivo de la elevación de ALT >400 U/L fue bajo $(27,7 \%)$, por lo que este indicador se muestra como poco fiable en el diagnóstico de lesión hepática aguda primaria. Deberían estudiarse otros marcadores séricos adicionales que evidencien dicha lesión.

Este estudio podría estar limitado por la heterogeneidad en edades de la población analizada, lo que podría estar afectando a la frecuencia relativa de determinadas etiologías. El periodo de tiempo analizado (2 años), aunque es superior al estudiado por otros autores $^{10,11}$, podría ser insuficiente para detectar determinadas etiologías de aparición infrecuente descritas en otros trabajos $^{11}$, como la colestasis gravídica. Futuras líneas de investigación deberían centrarse en el estudio etiológico de la HT marcada por grupos de edad, analizando además periodos de tiempo superiores.

\section{BIBLIOGRAFÍA}

1. Othmani S, Bahri M, Bahri M. Interpretation of hypertransaminasemia. Tunis Med. 2002; 80:23644.

2. Giannini EG, Testa R, Savarino V. Liver enzyme alteration: a guide for clinicians. CMAJ. 2005; 172:367-79.

3. Álvarez-Martínez H, Pérez-Campos E. El paciente con hipertransaminasemia. Rev Fac Med UNAM. 2005; 48:58-65.

4. Cuadrado A, Crespo J. Hipertransaminasemia en pacientes con negatividad de marcadores virales. Rev Esp Enferm Dig. 2004; 96:484-500.

5. Tietz NW, Rinker AD, Shaw LM. International Federation of Clinical Chemistry. IFCC methods for the measurement of catalytic concentration of enzymes. Part 5. IFCC method for alkaline phosphatase (orthophosphoric-monoester phosphohydrolase, alkaline optimum, EC 3.1.3.1). IFCC Document Stage 2, Draft 1, 1983-03 with a view 
to an IFCC Recommendation. Clin Chim Acta. 1983; 135:339F-67F.

6. Altman DG, Bland JM. Diagnostic tests 2: Predictive values. BMJ. 1994; 309:102.

7. Ibáñez H, Giménez A, Tomás S, Colomina J, Guerrero A. Ingresos hospitalarios por enfermedades infecciosas del aparato digestivo no intestinales. Enferm Infecc Microbiol Clin. 2006; 24 (Espec Congr):37-38.

8. Álvarez-Rocha L, Alós JI, Blanquer J, ÁlvarezLerma F, Garau J, Guerrero A, et al. Guía para el manejo de la neumonía comunitaria del adulto que precisa ingreso hospitalario. Med Intensiva. 2005; 29:21-62.

9. Sherlock S, Dooley J. Diseases of the liver. 11th ed. Oxford: Blackwell; 2002.

10. Whitehead MW, Hawkes ND, Hainsworth I, Kingham JG. A prospective study of the causes of notably raised aspartate aminotransferase of liver origin. Gut. 1999;45:129-33.
11. Bruguera M, Barrera JM, Corradi F, Mas A. Hipertransaminasemia superior a $400 \mathrm{U} / 1$ en adultos atendidos en un hospital terciario. Estudio prospectivo de su etiología. Gastroenterol Hepatol. 2005;28:15-9.

12. Sort P, Más A, Salmerón JM, Bruguera M, Rodés J. Recurrent liver involvement in heatstroke. Liver. 1996; 16:335-7.

13. Johnson RD, O’Connor ML, Kerr RM. Extreme serum elevations of aspartate aminotransferase. Am J Gastroenterol. 1995; 90:1244-5.

14. Vanejo I, Miranda ML, Stiefel P, Pamies E, Marenco ML, Castro D, et al. Características clinicobiológicas de un grupo de 54 gestantes con síndrome de HELLP. Med Clin (Barc). 2004; 122:259-61.

15. Tositti G, Fabris P, Romano L, De Lana F. Liver injury during gastroenteritis in adults: A retrospective study in 727 cases. Am J Gastroenterol. 2002; 97:487-8. 